\title{
How can we predict behavior of nanoparticles in vivo?
}

\author{
"Establishing a database that will contain nanoparticle properties, \\ protein corona fingerprints and corresponding biological outcomes \\ will enable maximizing the use of large datasets."
}

First draft submitted: 12 October 2015; Accepted for publication: 5 November 2015; Published online: 19 January 2016

Keywords: nanomedicine $\bullet$ nanoparticle biological identity $\bullet$ nanoparticles $\bullet$ protein corona - structure-activity models

The emergence of nanotechnology and nanoparticles has brought conceptually new possibilities for administration of therapeutic agents. Sustained release, targeting, altered pharmacokinetic, reduced toxicity and increased drug bioavailability make nanoscale drug delivery systems highly attractive for improving the therapeutic index of many drugs. It is quite striking that nanotechnology not simply represents a miniaturization of larger objects, but rather enables preparation of materials at the nanometric scale with physical and chemical properties which dramatically differ from those of bulk materials. The main reason for this remarkable change in nanomaterial behavior is its enormous surface-to-volume ratio, which provides a very large interfacial surface area as driving force for enhanced interaction of nanomaterial with surrounding it molecules. Therefore, upon intravenous administration, nanoparticles inevitably form layers of adsorbed biomolecules (mainly proteins) known as a 'protein corona' $[1,2]$.

The adsorption of proteins on the surface of nanoparticle is regulated by protein-nanoparticle binding affinity and by protein-protein interactions. Proteins that directly adsorb to the nanoparticle surface with high affinity (usually characterized by long desorption rates in the order of several hours [3]) form the first layer of tightly bound proteins known as the 'hard corona'. There is a second stage, which consists of proteins interacting with this firmly bound proteinnanoparticle complex via low-affinity protein-protein interactions, forming the socalled 'soft corona' and consisting of loosely bound proteins. The complex structure of protein corona alters the size and interfacial composition of nanoparticles, conferring them a new biological identity, which is what is 'seen' by cells in reality. The biological identity of nanoparticles, which could be significantly different from their original synthetic identity, determines the physiological behavior of nanoparticles influencing their colloidal stability, targeting capability, kinetics of circulation, transport, cellular uptake and organ accumulation, degradation, drug release, signaling and toxicity [4].

Because the relative quantities of the adsorbed proteins on the surface of nanoparticles do not necessarily correlate with their abundance in blood plasma, the composition of protein corona for each particular type of nanomaterial is unique and influenced by multiple factors. The complexity and uniqueness of the protein corona composition in each particular case of nanomaterial does not allow one to reliably predict behavior of nanomaterials in vivo, which remains one of the major challenges in achieving a predictable and safe use of nanoparticlebased drug delivery systems in therapeutic applications.

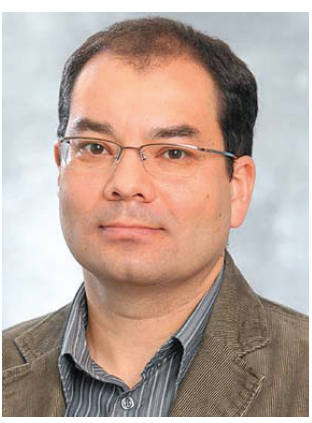

Boris Polyak

Author for correspondence: Laboratory of Magnetic Drug Targeting, Department of Surgery \& Department of Pharmacology \& Physiology, Drexel University, College of Medicine, Philadelphia, PA 19102, USA

Tel.: +1 2157623386

bpolyak@drexelmed.edu

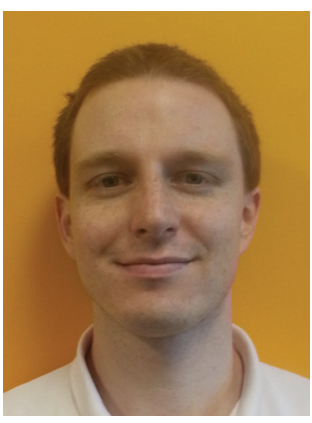

Bernardo Cordovez

Author for correspondence: Co-founder \& President, Optofluidics, Inc., Philadelphia, PA 19104, USA Tel.: +1 2159702683 bc@opfluid.com 
Commercially available analytical instrumentation as well as custom assays have been able to characterize particle drugs and the protein corona to important, but unfortunately not fully actionable degrees. As pioneering work from Dawson and many others have revealed, the formation of the corona is dictated by key parameters including core particle size, particle charge, temperature and proteins involved in the formation, suspension conditions, time and colloidal stability among other factors [5]. However, while current instrumentation and methodologies have produced important insights on the corona's physical structure and biochemical composition, there is a giant gap in how to use this in vitro information of basic particle parameters to predict the outcomes of these drugs in vivo.

So what has been measured? The thickness of this biological barrier has been measured using dynamic light scattering, ultracentrifugation, fluorescence correlation spectroscopy, single particle sizing tools and even other more complicated techniques including stained transmission electron microscopy imaging and UV-visible spectroscopy to measure agglomeration [6,7]. Dynamic light scattering, the work horse of particle analysis, provides useful and easy to generate particle size distribution information; but when it comes to measuring the corona thickness, this technique works only with essentially monodisperse particle distributions and faces issues measuring the corona thicknesses for particles greater than tens of nanometers (where the thickness change as a result of protein adsorption is much less than the particle core size itself). The presence of the corona can also be captured using charge measurements such as zeta potential to confirm the adsorption of proteins onto the carrier's surface. While useful and used as a standard measurement for corona formation $[3,8,9]$, zeta potential struggles with biologically complex samples and experience severe difficulty with the predominantly weakly negatively charged protein groups (low zeta).

Biochemical characterization of the protein corona consisted of various methodologies, including western blots, mass spectroscopy, surface plasmon resonance and others, which have been particularly useful in characterizing the hard corona (since this layer is not washed away during gel electrophoresis) [6,10-12]. As described above, great insights have been uncovered showing that the protein corona does not necessarily select for the most abundant proteins in serum $[13,14]$. The above only captures a small subset of the metrology work on protein corona measurements. As is well documented, all of these analysis techniques have their advantages and disadvantages and have painted a good starting picture of the corona, so what is missing?
We believe that there are three main obstacles that must be overcome so that metrology can help researchers understand and develop better particle-based drugs, given the ever present reality of the protein corona. First, there is a need for tools capable of measuring the weak interactions that are augmented in the nanoscale in high-throughput. Most systems are designed to detect strong interactions, like strong electrostatic forces, or require lots of material (bulk-based measurements) to operate. Weak interactions have been ignored or been out of the reach of most existing instrumentation. Second, particle analysis technologies that work more effectively in complex fluids like blood and serum under little dilution. This capability is a big challenge to systems optical and electronic alike, but chemical methods are fair better. Third, we have to challenge the idealistic concept of what we think the particle-based drugs look like after formulation versus what they really look like. There is a need to measure and validate what we claim.

To address these challenges, novel comprehensive surface characterization technologies have to be developed. For example, the Optofluidics NanoTweezer Surface Analysis System is designed to tackle the first and third challenge by enabling high-throughput colloidal surface analysis. Colloidal analysis is in dire need of greater surface characterization since the surface is the vehicle of interaction for these particles, it is where the corona is formed and where it changes with the environment and time, but such measurements have been largely disregarded by current analytical techniques. To measure surface properties, including the corona and other surface coatings, the system captures the total interaction potential of weak and strong interactions (the summation of the electrostatic, hydrogen bonding, steric and other forces) at the individual nanoparticle level [15] through the use of microfluidics and nanophotonic waveguides. Its outputs include particle repulsion strength and characteristic interaction length, and maps how the particles interact against different reference surfaces, allowing one to screen for specific interactions. Greater insights of these weak interactions can enable better understanding of corona formation and dissolution, for example, the steric contribution in protein-protein interactions present in the corona, yielding information key to understanding the biophysical basis, and one step closer to answering which forces are involved in determining whether a targeted nanoparticle is cleared by the immune system of reach the target tumor site. Also, by being able to measure subpopulations (one particle at a time), one can start to assess surface heterogeneity and examine whether all particles exhibit the same surface characteristics (e.g., are all my particles coated with PEG and how uniform is that protein corona?). 
In addition to the advancement of novel analytical techniques enabling high-throughput colloidal surface analysis and investigation of protein corona composition, quantitative models capable to accurately predict biological interactions of nanoparticles in physiological milieu are needed to accelerate clinical translation of nanoparticle-based drugs. Recently, Chan and coworkers have proposed a novel approach to predict the cellular interaction of gold and silver nanoparticles based on quantitative structure-activity relationships, which link the composition of the formed protein corona with the bioactivity of nanoparticles [14]. The composition of the protein corona 'fingerprint' (based on the relative abundance of the serum proteins adsorbed on the nanoparticle surface) was used to predict the cell association of about a hundred-member library of chemically and physically diverse nanoparticles. Cell association (nanoparticle surface binding and uptake) was chosen as a model biological interaction due to its relevance to inflammatory response, biodistribution and toxicity. This work has shown that the quantitative model that uses the protein corona composition fingerprint was 50\% more accurate than a model that uses physicochemical properties of nanoparticles such as size, aggregation state and surface charge [14]. While the above work has demonstrated that protein corona fingerprints contribute more significant information than the physicochemical properties to make an accurate prediction of biological behavior of nanoparticles, the overall prediction can be further improved if the model will use both corona composition and relevant physicochemical properties [16].

It is of note, that blood serum does not comprise an ideal model reflecting a realistic in vivo environment, because key blood coagulation factors are absent. To make more accurate predictions for nanoparticles behavior in vivo, the future correlation models may consider using nanoparticles exposed to a whole blood or even injected into the blood stream with subsequent isolation and characterization that will reflect a more realistic corona composition. This strategy may contribute to our understanding whether there is a dramatic difference in corona composition when particles exposed to real conditions in vivo and how this affects nanoparticle biological behavior and fate.

Using the quantitative structure-activity relationships that will link the information obtained by the

\section{References}

1 Lynch I, Salvati A, Dawson KA. Protein-nanoparticle interactions: what does the cell see? Nat. Nanotechnol. 4(9), 546-547 (2009).

2 Rahman M, Laurent S, Tawil N, Yahia L, Mahmoudi M. Protein-nanoparticle interactions. In: Biophysics 15. SpringerVerlag, Berlin, Heidelberg, Germany (2013). most advanced colloidal surface analysis techniques with the protein corona composition data (originated from ideal in vivo conditions) and corresponding biological outcomes, the researchers will be able to make more accurate predictions of nanoparticles association with various relevant cells types, such as circulating immune cells, endothelial cells (lining the lumen of the blood vessels) and organ-related hepatocytes and splenocytes. Because the nanoparticle-cell interactions influence multiple downstream cellular responses, the predictions could be extended to characterize signaling pathways, cytokine secretion, gene expression and toxicity. This knowledge can be further extended to predict the realistic in vivo pharmacokinetics, biodistribution and organ response. Using the recently proposed alphanumeric nomenclature system, nanomaterials possessing specific chemical and physical properties can now be categorized and indexed [17]. Establishing a database that will contain nanoparticle properties, protein corona fingerprints and corresponding biological outcomes will enable maximizing the use of large datasets [14]. Ultimately, such databases will allow the broader research community to model biological interactions without the need for extensive experimentation, which will lead to establishment of predictive models that will guide the design of nanoparticle-based systems for diverse therapeutic applications.

\section{Disclaimer}

The content is solely the responsibility of the authors and does not necessarily represent the official views of the National Heart, Lung and Blood Institute or the $\mathrm{NIH}$.

\section{Financial \& competing interests disclosure}

This work was supported by the National Heart, Lung and Blood Institute (award number R01HL107771) and the Drexel University College of Medicine Clinical \& Translational Research Institute (CTRI) grant to B Polyak. B Cordovez has financial interest in Optofluidics, Inc. The authors have no other relevant affiliations or financial involvement with any organization or entity with a financial interest in or financial conflict with the subject matter or materials discussed in the manuscript apart from those disclosed.

No writing assistance was utilized in the production of this manuscript.

3 Monopoli MP, Walczyk D, Campbell A et al. Physicalchemical aspects of protein corona: relevance to in vitro and in vivo biological impacts of nanoparticles. J. Am. Chem. Soc. 133(8), 2525-2534 (2011).

4 Brun E, Sicard-Roselli C. Could nanoparticle corona characterization help for biological consequence prediction? Cancer Nanotechnol. 5(1), 7 (2014). 
5 Del Pino P, Pelaz B, Zhang Q, Maffre P, Nienhaus GU, Parak WJ. Protein corona formation around nanoparticles from the past to the future. Materials Horizons 1(3), 301-313 (2014).

6 Cedervall $\mathrm{T}$, Lynch I, Lindman $\mathrm{S}$ et al. Understanding the nanoparticle-protein corona using methods to quantify exchange rates and affinities of proteins for nanoparticles. Proc. Natl Acad. Sci. USA 104(7), 2050-2055 (2007).

7 Tenzer S, Docter D, Rosfa $S$ et al. Nanoparticle size is a critical physicochemical determinant of the human blood plasma corona: a comprehensive quantitative proteomic analysis. ACS Nano 5(9), 7155-7167 (2011).

8 Casals E, Pfaller T, Duschl A, Oostingh GJ, Puntes V. Time evolution of the nanoparticle protein corona. ACS Nano 4(7), 3623-3632 (2010).

9 Monopoli MP, Pitek AS, Lynch I, Dawson KA. Formation and characterization of the nanoparticle-protein corona. Methods Mol. Biol. 1025 137-155 (2013).

10 Jiang X, Weise S, Hafner M et al. Quantitative analysis of the protein corona on FePt nanoparticles formed by transferrin binding. J. R. Soc, Interface 7(Suppl. 1), S5-S13 (2010).

11 Lundqvist M, Stigler J, Elia G, Lynch I, Cedervall T, Dawson KA. Nanoparticle size and surface properties determine the protein corona with possible implications for biological impacts. Proc. Natl Acad. Sci. USA 105(38), 14265-14270 (2008).

12 Tassa C, Duffner JL, Lewis TA et al. Binding affinity and kinetic analysis of targeted small molecule-modified nanoparticles. Bioconjug. Chem. 21(1), 14-19 (2010).

13 Walkey CD, Olsen JB, Guo H, Emili A, Chan WC. Nanoparticle size and surface chemistry determine serum protein adsorption and macrophage uptake. J. Am. Chem. Soc. 134(4), 2139-2147 (2012).

14 Walkey CD, Olsen JB, Song F et al. Protein corona fingerprinting predicts the cellular interaction of gold and silver nanoparticles. ACS Nano 8(3), 2439-2455 (2014).

15 Schein P, Kang P, O'dell D, Erickson D. Nanophotonic force microscopy: characterizing particle-surface interactions using near-field photonics. Nano Lett. 15(2), 1414-1420 (2015).

16 Liu R, Jiang W, Walkey CD, Chan WC, Cohen Y. Prediction of nanoparticles-cell association based on corona proteins and physicochemical properties. Nanoscale 7(21), 9664-9675 (2015).

17 Gentleman DJ, Chan WC. A systematic nomenclature for codifying engineered nanostructures. Small 5(4), 426-431 (2009). 